\title{
Tinnitus with a Normal Audiogram: Physiological Evidence for Hidden Hearing Loss and Computational Model
}

\author{
Roland Schaette and David McAlpine \\ University College London Ear Institute, London WC1X 8EE, United Kingdom
}

Ever since Pliny the Elder coined the term tinnitus, the perception of sound in the absence of an external sound source has remained enigmatic. Traditional theories assume that tinnitus is triggered by cochlear damage, but many tinnitus patients present with a normal audiogram, i.e., with no direct signs of cochlear damage. Here, we report that in human subjects with tinnitus and a normal audiogram, auditory brainstem responses show a significantly reduced amplitude of the wave I potential (generated by primary auditory nerve fibers) but normal amplitudes of the more centrally generated wave V. This provides direct physiological evidence of "hidden hearing loss" that manifests as reduced neural output from the cochlea, and consequent renormalization of neuronal response magnitude within the brainstem. Employing an established computational model, we demonstrate how tinnitus could arise from a homeostatic response of neurons in the central auditory system to reduced auditory nerve input in the absence of elevated hearing thresholds.

\section{Introduction}

Tinnitus is a frequent phenomenon occurring in an estimated $10-15 \%$ of the population (Hoffman and Reed, 2004; Henry et al., 2005). In 1-2\% of the population tinnitus symptoms seriously reduce quality of life, resulting in social isolation, depression, and even suicidal tendencies. The first usage of the term "tinnitus" for ringing in the ears has been attributed to Pliny the Elder (Morgenstern, 2005), and written descriptions of tinnitus remedies date back to ancient Egypt (Stephens, 1984). However, causal therapeutic interventions in tinnitus are virtually nonexistent; sound maskers, tinnitus-retraining therapy, and psychologically inspired interventions such as cognitive behavioral therapy are aimed primarily at alleviating the symptoms and distress caused by tinnitus.

Development of treatments to eliminate rather than just alleviate tinnitus and identification of factors that cause this often debilitating condition would be greatly advanced if the neural origins of tinnitus were established. To this end, tinnitus patients with apparently normal hearing (Barnea et al., 1990; Sanchez et al., 2005) represent a challenge to models of tinnitus generation that rely on compromised cochlear function to elicit hyperactivity in brain structures (Schaette and Kempter, 2006; Parra and Pearlmutter, 2007; Kaltenbach, 2010; Rauschecker et al., 2010; Roberts et al., 2010). Cochlear damage is normally manifest as an elevation in hearing thresholds-assessed through pure-tone au-

\footnotetext{
Received April 30, 2011; revised July 13, 2011; accepted July 25, 2011.

Author contributions: R.S. and D.M. designed research; R.S. performed research; R.S. analyzed data; R.S. and D.M. wrote the paper.

This study was supported by the British Tinnitus Association. We thank Paul Radomskij for helpful discussions about the ABR experiments, Thomas Cracknell and Leon Cox for assistance in the measurements, Lucy Anderson for blind analysis of the ABR data, and Jennifer Linden for valuable comments on the manuscript.

The authors have no competing financial interests.

Correspondence should be addressed to Roland Schaette, UCL Ear Institute, 332 Gray's Inn Road, London WC1X 8EE, United Kingdom. E-mail: r.schaette@ucl.ac.uk.

DOI:10.1523/JNEUROSCI.2156-11.2011

Copyright $\odot 2011$ the authors $\quad 0270-6474 / 11 / 3113452-06 \$ 15.00 / 0$
}

diometry - and the absence of any detectable loss of cochlear function in these individuals has been taken to indicate that tinnitus can arise without any peripheral hearing loss.

However, in a recent demonstration that normal hearing thresholds do not necessarily indicate absence of cochlear damage, mice subjected to mild acoustic trauma displayed a temporary shift in hearing thresholds but a permanent deafferentation of some $50-60 \%$ of the auditory nerve (AN) fibers in the highfrequency region of the cochlea (Kujawa and Liberman, 2009). This suggests that deafferentation following noise damage predominantly affects high-threshold AN fibers, while sufficient numbers of low-threshold AN fibers remain responsive to sound. Normal hearing thresholds can also be accompanied by impaired function of efferent fibers that project from the brainstem to the cochlea (Kim et al., 2002; Jacobson et al., 2003; Zettel et al., 2007; Zhu et al., 2007).

Based on computational models of tinnitus development (Dominguez et al., 2006; Schaette and Kempter, 2006, 2009), we hypothesized that deafferentation of a substantial fraction of the AN fibers, as observed in mice following "temporary" hearing loss (Kujawa and Liberman, 2009), could trigger the development of a neural correlate of tinnitus in central auditory structures.

Here, we demonstrate in subjects with tinnitus and apparently normal hearing a deficit in AN function manifested as a reduction in nerve output at high sound levels, indicating deafferentation of high-threshold AN fibers. This deficit appears to be compensated for at the level of the brainstem, supporting the view that tinnitus is promoted by homeostatic mechanisms that act to normalize levels of neural activity in the central auditory system.

\section{Materials and Methods}

This study was approved by the University College London (UCL) ethics committee. Participants were recruited through an e-mail advertisement to UCL staff and students. Thirty-three female subject, fifteen with tinnitus (mean age $36.3 \pm 2.6$ years) and eighteen controls (mean age $33.2 \pm$ 
1.9 years, no significant age difference, $p=0.32, t$ test), participated in this study. Due to gender differences in the magnitude of auditoryevoked potentials (Durrant et al., 1990), we studied only female participants. Tinnitus participants were required to have chronic tinnitus, i.e., of $>6$ months duration and a stable, nonpulsatile tinnitus percept. Audiograms were measured with a calibrated clinical audiometer (Kamplex KC 50, with Telephonics TDH 49 headphones for $0.125-8 \mathrm{kHz}$, and Koss $\mathrm{R} / 80$ headphones for 12 and $16 \mathrm{kHz}$ ). Tinnitus was characterized using a modified tinnitus spectrum procedure (Norena et al., 2002): subjects matched pure tones $(0.25-16 \mathrm{kHz})$ to the loudness of their tinnitus and rated the similarity in pitch of tones and their tinnitus on a scale from 0 to 10 (three presentations per tone, in random order). Mean tinnitus spectra were calculated by averaging ratings across participants for each frequency. Comparison tones were generated by a computer with custom-made software and an ASUS Xonar Essence ST sound card and presented via Sennheiser HD600 headphones.

Auditory brainstem responses were measured using a Medelec Synergy T-EP system (Oxford Instruments Medical). Disposable electrodes (Nicolet Biomedical) were placed on the high forehead and the ipsilateral and contralateral mastoids. Electrode impedances were $<2 \mathrm{k} \Omega$. Stimuli were $50 \mu$ s clicks at 90 and $100 \mathrm{~dB}$ sound pressure level (SPL) (peak values) presented via Telephonics TDH 49 headphones at a rate of 11 clicks/s. Signals were bandpass filtered $(100-1500 \mathrm{~Hz})$ and averaged ( $\geq 8000$ repetitions for $90 \mathrm{~dB} \mathrm{SPL}, \geq 6000$ repetitions for $100 \mathrm{~dB} \mathrm{SPL}$ ). Amplitudes of wave I and $\mathrm{V}$ were measured from peak to following trough.

In a computational model of the first stages of auditory processing (Schaette and Kempter, 2006, 2009), deafferentation of high-threshold AN fibers (as observed in mice following temporary noise-induced hearing loss) was modeled by decreasing the slope of the AN fiber population response $f(I)$ for high intensities:

$$
f^{\star}(I)=f(I)-d\left[f(I)-f_{d}\right]_{+},
$$

where $I$ is the intensity of the acoustic stimulus, $d$ controls the degree of deafferentation, $f_{\mathrm{d}}=75 \mathrm{sp} / \mathrm{s}$ determines the level where deafferentation starts influencing AN responses, and [ $]_{+}$denotes positive rectification. $f(I)$ represents the average firing rate of a small population of AN fibers with similar characteristic frequencies. As in a previous version of the model, the spontaneous firing rate of the AN fiber population (an average over all AN fiber types, thus comprising high-spontaneous as well as low-spontaneous rate fibers) was set to $f_{\mathrm{sp}}=50 \mathrm{spikes} / \mathrm{s}$, the maximum firing rate to $f_{\max }=250 \mathrm{sp} / \mathrm{s}$, and the response threshold to $I_{\text {th }}=0 \mathrm{~dB}$, corresponding to the threshold of the most sensitive AN fibers. Resulting rate-intensity functions are shown in Figure $3 b$.

Assuming a Gaussian probability distribution for sound intensities (in units of $\mathrm{dB}$ ) in the acoustic environment [ $40 \mathrm{~dB}$ mean intensity, $25 \mathrm{~dB}$ standard deviation; see Schaette and Kempter (2006) for more details], we calculated how deafferentation changes the mean activity of the AN and the central auditory neurons in the model.

For the cochlear nucleus stage of the model we employed a parameter set that yields type III response characteristics in the principal neurons, as neurons of this model type are suggested to become hyperactive after hearing thresholds increase (Schaette and Kempter, 2008). Homeostatic plasticity was implemented in the model through a homeostasis factor $h$, which scales, in opposite directions, the strength of excitatory and inhibitory synapses onto cochlear nucleus $(\mathrm{CN})$ principal neurons. The exact value of $h$ required to restore the mean activity to healthy target levels was determined numerically [see Schaette and Kempter (2008)].

To relate the results obtained with the tinnitus model to the auditory brainstem response (ABR) data, we simulated $A B R$ wave I using the MATLAB model of the auditory periphery (MAP) (Meddis, 2006). We considered a population of $600 \mathrm{AN}$ fibers with characteristic frequencies from 2 to $16 \mathrm{kHz}$ in 0.1 octave steps. By varying the parameter $\tau_{\mathrm{Ca}}$ of the MAP model from 0.35 to $0.072 \mathrm{~ms}$ in 19 steps, reducing $\tau_{\mathrm{Ca}}$ by $8 \%$ for each step, we produced 20 different AN fiber types from low-threshold/ high spontaneous-rate fibers to high-threshold/low spontaneous-rate fibers. Fiber thresholds ranged from 0 to $62 \mathrm{~dB}$ SPL with a mean threshold of $16.5 \mathrm{~dB}$. The MAP model was stimulated with a $50 \mu$ s click at $90 \mathrm{~dB}$
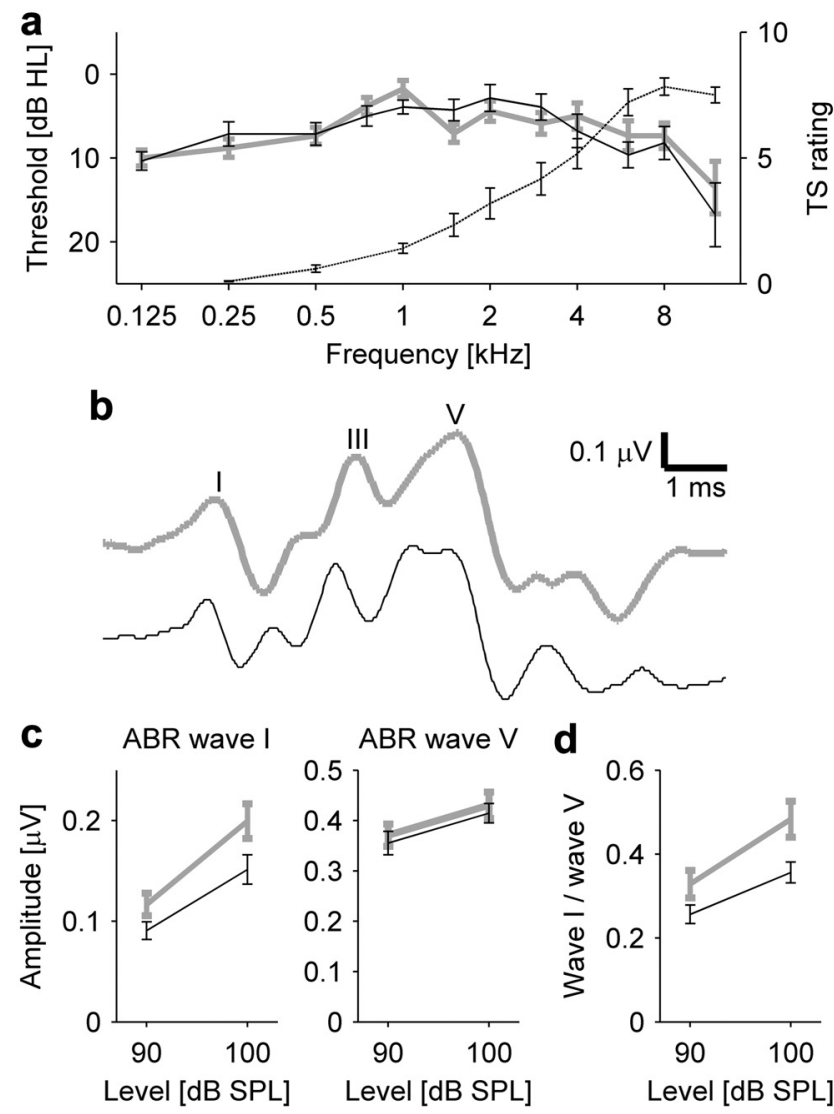

Figure 1. Auditory brainstem responses in tinnitus with normal hearing thresholds. $\boldsymbol{a}$, Mean audiogram (black line) and mean tinnitus spectrum (dotted line) of the tinnitus group ( $n=15$ ) and mean audiogram of the control group (gray line, $n=18$ ). TS, Tinnitus spectrum. $\boldsymbol{b}$, Example $A B R$ waveform of a tinnitus (black line) and a control subject (gray line) for $50 \mu$ s clicks at $100 \mathrm{~dB}$ SPL. The roman numerals label waves I, III, and V of the ABR. C, Mean amplitudes of ABR wave I are significantly lower in the tinnitus (black) than in the control group (gray, $p=0.009$, twoway ANOVA), whereas amplitudes of wave V do not differ significantly. $\boldsymbol{d}$, Wave I amplitude normalized by wave $V$ amplitude also shows a significant difference between tinnitus and control ( $p=0.004$, two-way ANOVA).

SPL. ABR wave I was obtained by convolving each action potential produced by each AN fiber with a spike waveform and then summing across all AN fibers.

Simulations of the tinnitus model and the MAP model and all data analyses were performed using MATLAB (MathWorks). Differences in age and hearing thresholds were assessed using two-sample $t$ tests. To test for significant differences in ABR wave amplitudes, we used two-way ANOVA with group and level as factors. All errors are given as \pm SEM.

\section{Results}

Thirty-three female subjects, fifteen with tinnitus and eighteen controls, participated in this study. All participants had normal hearing (hearing thresholds $\leq 20 \mathrm{~dB}$ hearing level (HL) from 125 $\mathrm{Hz}$ to $8 \mathrm{kHz}$ ), and there was no significant difference in the average hearing thresholds up to $12 \mathrm{kHz}$ (Fig. 1 $a$; black line, tinnitus; gray line, control; all $p>0.05, t$ test). Five participants in the tinnitus and three in the control group were unable to hear 16 $\mathrm{kHz}$. Measurements of tinnitus spectra within the tinnitus group (see Materials and Methods) indicate that comparison sounds $\geq 6 \mathrm{kHz}$ were rated as most similar to the pitch of the tinnitus (see Fig. $1 a$, dotted line, for average tinnitus spectrum).

To test for "hidden hearing loss," ABRs were measured with $50 \mu$ s clicks at 90 and $100 \mathrm{~dB}$ SPL. The mean amplitude of wave I of the ABR (Fig. 1c), reflecting the summed response to sound of 
the primary afferent nerve fibers innervating the inner hair cells of the cochlea (Moller, 2007) (see Fig. 2 for illustration), was significantly smaller in the tinnitus group than in the control group (90 dB: $0.091 \pm 0.009 \mu \mathrm{V}$ vs $0.121 \pm 0.012 \mu \mathrm{V}$; $100 \mathrm{~dB}: 0.151 \pm 0.015 \mu \mathrm{V}$ vs $0.203 \pm$ $0.017 \mu \mathrm{V} ; p=0.009$, two-way ANOVA). This suggests either a reduced number of responsive AN fibers (e.g., due to deafferentation; Fig. $2 b$ ), reduced synchrony in the discharge of the AN fibers, or both. Similar results have been reported in mice subjected to mild acoustic trauma and in which deafferentation of a large fraction of AN fibers occurs without permanent changes in hearing thresholds (Kujawa and Liberman, 2009). In contrast to wave $\mathrm{I}$, the amplitude of wave $\mathrm{V}$ of the ABR, generated at the level of the auditory midbrain (Moller, 2007) (see Fig. 2 for illustration), did not differ significantly between the tinnitus and nontinnitus groups ( $p=0.50$, two-way ANOVA; Fig. $1 c)$, suggesting that homeostatic mechanisms in central auditory structures adjust neural responsiveness to compensate for reduced input from the AN (Fig. 2b).

To determine how deafferentation of AN fibers might lead to increased central gain and the generation of tinnitus, we employed a computational model of the early stages of auditory processing (Fig. $3 a$ ). Previously, this model was used to demonstrate how hearing loss (i.e., increases in hearing thresholds) might lead to the development of increased spontaneous neuronal activity as observed in animal models of tinnitus (Schaette and Kempter, 2006, 2008); its predictions of tinnitus frequencies from audiograms closely match perceived tinnitus pitch (Schaette and Kempter, 2009). In the model, the development of neural correlates of tinnitus constitutes a side effect of the stabilization of mean activity in central auditory neurons through a mechanism of homeostatic plasticity: hearing loss reduces AN activity with a concomitant reduction in excitatory drive to the central auditory system. To stabilize mean neuronal activity, homeostatic mechanisms generate increased excitatory gain and reduced inhibitory gain in neurons downstream of the auditory nerve, restoring average neuronal activity to normal levels. However, as neurons become more excitable, spontaneous activity is amplified, leading to hyperactivity and the generation of a tinnitus percept.

In the model, we adapted the AN stage to account for a deafferentation of AN fibers by introducing an additional parameter that controls the shape of the auditory nerve rate-versus-intensity functions (Fig. $3 b$ and Materials and Methods). All parameters of the model relating to the central auditory pathways remained unchanged. In the AN model, we assumed that the most sensitive AN fibers remain undamaged, a necessary requirement for preserving
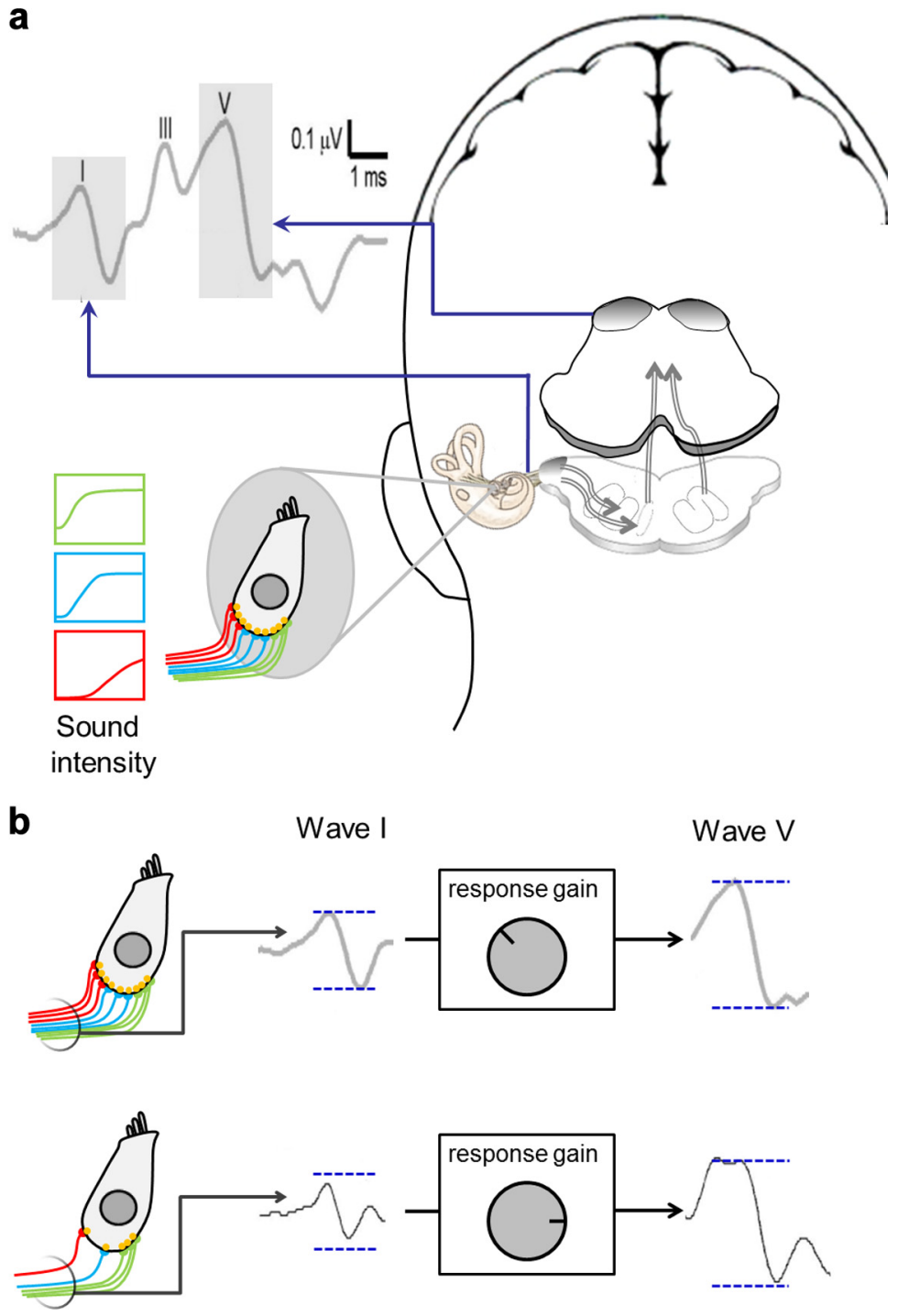

Figure 2. Auditory brainstem responses, hidden hearing loss, and homeostatic gain control in the auditory system. $\boldsymbol{a}$, Illustration of the generation sites of wave I (auditory nerve) and wave V (midbrain) of the ABR, schematic depiction of an inner hair cell 作 could normalize wave V amplitude after hidden hearing loss. In the healthy situation (top),

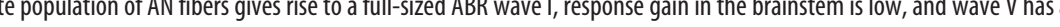
normal amplitude. In hidden hearing loss (bottom), a fraction of the AN fibers no longer responds to sound, leading to a reduced amplitude of ABR wave I; but through increased response gain, the amplitude of wave $V$ has been restored to a normal size.

normal hearing thresholds in quiet and consistent with the effects of mild acoustic trauma in mice (Kujawa and Liberman, 2009). Deafferentation of AN fibers reduced mean activity in both the AN (Fig. 3c) and the central auditory system (Fig. 3d, gray line). When homeostatic plasticity restored mean activity to its healthy target level (Fig. $3 d$, black line), the resulting increase in neuronal response gain led to an amplification of spontaneous activity and neurons became "hyperactive" (Fig. 3e). Thus, in the model, a reduction of AN responses, caused by deafferentation of AN fibers at the hair cell synapses, leads to the development of a neural correlate of tinnitus; tinnitus-related hyperactivity is generated because the response gain of central neuronal circuits is pathologically increased (Fig. $4 b$ ). This prediction matches the increased central gain observed in the ABR wave $\mathrm{V}$ of our tinnitus subjects (Fig. 4a).

To compare the model outcome and human data more directly, we first determined the relative increase in central re- 
a

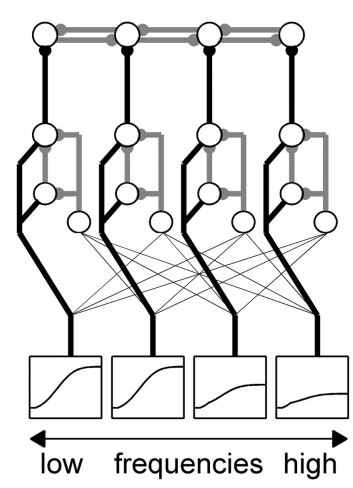

b

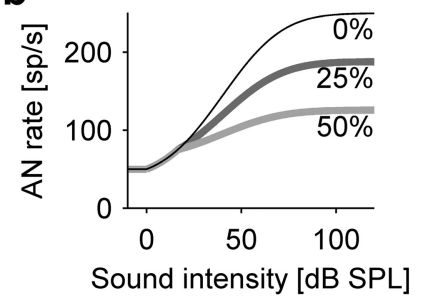

C
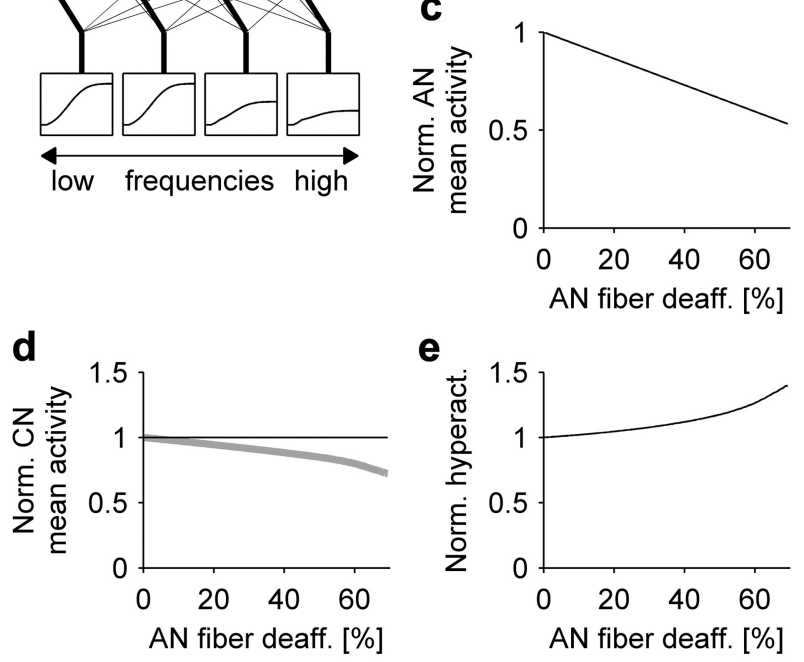

e

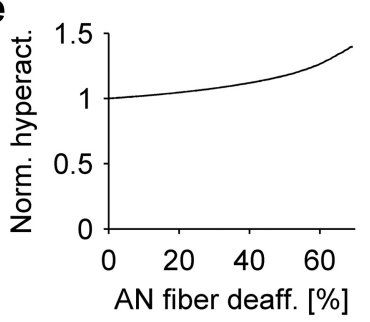

Figure 3. A computational model demonstrates how AN fiber deafferentation (deaff.) could lead to tinnitus-related neuronal hyperactivity. $\boldsymbol{a}$, Architecture of the model covering auditory nerve (bottom), cochlear nucleus (middle), and a central processing stage with lateral inhibition (top); four frequency channels are shown. Circles denote neurons, black lines excitatory connections, and gray lines inhibitory connections. $\boldsymbol{b}$, Model AN rate-versus-intensity functions are scaled down to account for deafferentation of AN fibers. sp/s, Spikes per second. $c$, The mean AN activity is reduced in proportion to the degree of deafferentation. Norm., Normalized. d, Mean activity in the $\mathrm{CN}$ stage of the model before (gray line) and after activity stabilization through homeostatic plasticity. e, Increased spontaneous firing rates (hyperactivity) as a side effect of activity stabilization.

sponse gain in our tinnitus subjects by calculating their average ratio of $A B R$ wave $V$ to wave $I$, normalized by the average ratios from the control group. For sound levels of $90 \mathrm{~dB}$ SPL we obtained a relative gain of 1.20, and 1.27 for $100 \mathrm{~dB}$ SPL (Fig. 4a). In the model, increases in excitatory gain of this magnitude were observed for 53 and $61 \%$ AN fiber deafferentation, respectively (Fig. $4 b$ ). To test whether such cochlear damage could underlie the generation of tinnitus, we then performed a simulation in which the mean audiogram of the tinnitus group was combined with a pattern of $60 \%$ AN fiber deafferentation in the highfrequency range $(>4 \mathrm{kHz})$. The model developed a hyperactivity pattern that was consistent with high-pitched tinnitus as observed in the tinnitus spectra measured in our tinnitus participants (Fig. 4c). Finally, using the MATLAB model of the auditory periphery (MAP; Meddis, 2006) — a detailed phenomenological model of cochlear and AN responses-we simulated ABR wave I for $50 \mu$ s clicks at $90 \mathrm{~dB}$ SPL for a normal cochlea and for a cochlea with the pattern of AN fiber deafferentation employed in Figure $4 c$. In the MAP model simulations, the amplitude of wave I from the damaged cochlea (black line) was reduced by $22 \%$ compared to the normal cochlea (gray line). For comparison, in the human tinnitus group, wave I amplitude was reduced on average by $22 \%$ for $90 \mathrm{~dB}$ SPL and by $24 \%$ for $100 \mathrm{~dB}$ SPL. Thus, cochlear damage that, in the tinnitus model, leads to a neuronal hyperactivity pattern consistent with the characteristics

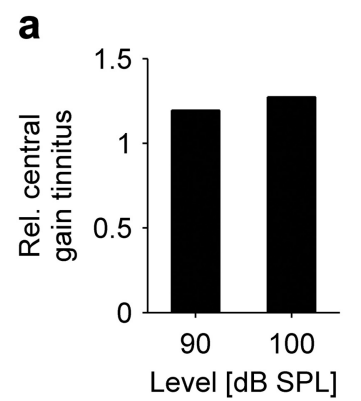

C

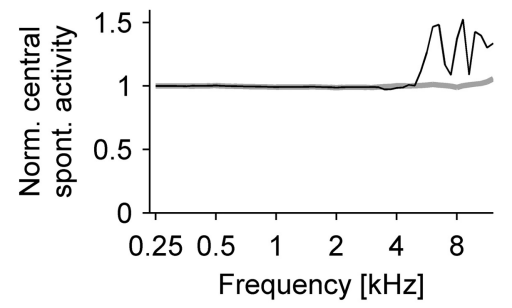

b

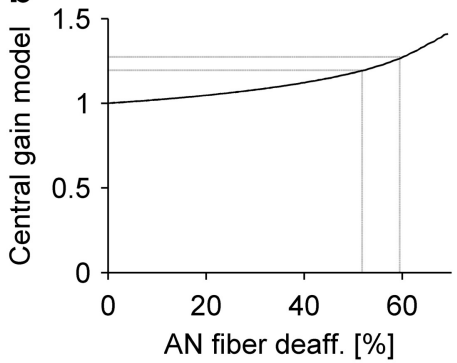

d

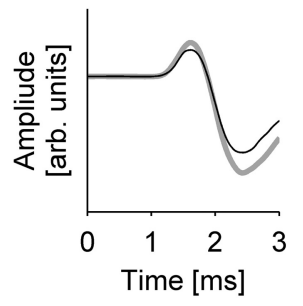

Figure 4. Relating measurements and model. $\boldsymbol{a}$, Central gain (mean ratio of ABR wave V/I) in tinnitus subjects normalized by control subjects. Rel., Relative. $\boldsymbol{b}$, Model results for the increase in central excitatory gain through homeostatic plasticity. The dotted lines indicate that gain increases as observed in tinnitus were observed in the model for 53 and $61 \%$ AN fiber deafferentation. c, Profile of spontaneous activity in the lateral-inhibition layer of the model for $\sim 60 \%$ AN fiber deafferentation above $4 \mathrm{kHz}$ before (gray line) and after homeostasis (black line). Norm., Normal. $\boldsymbol{d}$, Simulated ABR wave I for a normal cochlea (gray line) and for deafferentation of $60 \%$ of the AN fibers above $4 \mathrm{kHz}$ (black line), which reduces wave I amplitude by $22 \%$. arb., Arbitrary.

of tinnitus, also provides a close match with the reduction in the magnitude of ABR wave I in tinnitus subjects.

\section{Discussion}

Our finding of a reduced amplitude of ABR wave I in subjects with tinnitus resembles findings from mice where decreased wave I amplitudes, following temporary hearing threshold shifts, were linked to deafferentation of AN fibers (Kujawa and Liberman, 2009). Whether these mice also experienced tinnitus is unknown. However, in rats degeneration of AN fibers has been linked to behavioral evidence for tinnitus (Bauer et al., 2007), although these rats also suffered permanent hearing loss in the form of elevated hearing thresholds in quiet. Similarly, increased spontaneous neuronal activity has been observed in the inferior colliculus of guinea pigs (Mulders and Robertson, 2009) and in the dorsal cochlear nucleus of hamsters (Finlayson and Kaltenbach, 2009) after mild acoustic trauma that resulted in only limited hearing loss. These findings are generally consistent with our experimental data and the model predictions. A critical mechanism in our model, homeostatic plasticity, is thought to stabilize the mean activity of neurons on long time scales (Turrigiano, 1999). In response to activity deprivation, the strength of excitatory synapses is scaled up (Turrigiano et al., 1998), the strength of inhibitory synapses scaled down (Kilman et al., 2002), and intrinsic excitability of neurons is increased (Desai et al., 1999). These changes could lead to hyperexcitable neuronal networks that amplify spontaneous activity (Houweling et al., 2005). After hearing loss, increases in excitation and decreases in inhibition have been observed at various stages of the auditory system, including the auditory cortex (Kotak et al., 2005), the inferior colliculus (Vale and Sanes, 2002), and the cochlear nucleus (Whiting et al., 2009). Thus, homeostatic plasticity constitutes a reasonable 
candidate mechanism for generating tinnitus-related neuronal hyperactivity in response to reduced AN output.

Derived band ABR measurements show that wave I of the click-evoked ABR I is mainly generated by neurons with characteristic frequencies above $2 \mathrm{kHz}$ (Don and Eggermont, 1978); therefore, click-evoked ABR measurements can be used to screen the most common range of tinnitus frequencies (König et al., 2006) for the presence of cochlear damage. Wave $V$, on the other hand, can also be evoked by lower frequencies (Don and Eggermont, 1978). However, in the derived-band ABR, wave V in response to $1 \mathrm{kHz}$ and below appears later than the average latency of even the trough of wave $\mathrm{V}$ in our click-evoked ABR data (6.62 $\mathrm{ms}$ in the control and $6.65 \mathrm{~ms}$ in the tinnitus group). Thus, in our measurements, wave $\mathrm{V}$ might rather reflect contributions from high frequencies similar to wave I, making it possible to directly relate the amplitudes of wave I and wave $\mathrm{V}$ to assess neuronal response gain in the brainstem.

It has been demonstrated previously that tinnitus patients with normal audiograms have increased tone detection thresholds in high-intensity background noise (Weisz et al., 2006), and this psychophysical evidence for a processing deficit is now corroborated by our data showing reduced AN output at high intensities. Our results indicate deafferentation of high-threshold AN fibers, a form of cochlear damage consistent with increased tone detection thresholds in background noise. Additionally, reduced feedback from the medial olivocochlear system might also contribute to increased tone detection thresholds in noise. Thus, future investigations could exploit the contralateral suppression of otoacoustic emissions to examine putative links between tinnitus and the olivocochlear system.

In addition to providing physiological evidence for deficits in cochlear processing in tinnitus patients with a normal audiogram, our results go one crucial step further by demonstrating that the putative deafferentation of AN fibers is associated with increased neural gain at the level of the brainstem. This suggests a potential mechanism for the generation of tinnitus. It remains unclear where in the auditory system tinnitus is first generated, as putative neural correlates have been observed at all stages of the central pathways (Roberts et al., 2010). A recent hypothesis assumes that pathological changes in spontaneous neuronal activity in the auditory brainstem could drive reorganization of auditory cortex and that conscious perception of tinnitus only occurs after both processes have taken place (Rauschecker et al., 2010). In rats, the degree of reorganization of the auditory cortex is correlated with the strength of behavioral evidence for tinnitus (Engineer et al., 2011), supporting an involvement of cortical structures. Our model demonstrates how activity-dependent neuronal plasticity in response to hidden hearing loss could lead to pathological activity patterns in the auditory brainstem that potentially trigger the development of tinnitus. The model also shows that the same mechanism of activity stabilization through homeostatic plasticity in the central auditory system can explain the development of such a neural substrate of tinnitus with (Schaette and Kempter, 2009) and without apparent hearing loss, thus presenting a unifying mechanism for the initiation of tinnitus. If the initiation site of tinnitus is located in the auditory brainstem, as our data, our model, and animal models of tinnitus (Mulders and Robertson, 2009; Kaltenbach, 2010) indicate, then brainstem structures might be targeted to develop specific drug treatments for this often debilitating condition.

\section{References}

Barnea G, Attias J, Gold S, Shahar A (1990) Tinnitus with normal hearing sensitivity: extended high-frequency audiometry and auditory-nerve brain-stem-evoked responses. Audiology 29:36-45.

Bauer CA, Brozoski TJ, Myers K (2007) Primary afferent dendrite degeneration as a cause of tinnitus. J Neurosci Res 85:1489-1498.

Desai NS, Rutherford LC, Turrigiano GG (1999) Plasticity in the intrinsic excitability of cortical pyramidal neurons. Nat Neurosci 2:515-520.

Dominguez M, Becker S, Bruce I, Read H (2006) A spiking neuron model of cortical correlates of sensorineural hearing loss: Spontaneous firing, synchrony, and tinnitus. Neural Comput 18:2942-2958.

Don M, Eggermont JJ (1978) Analysis of the click-evoked brainstem potentials in man using high-pass noise masking. J Acoust Soc Am 63:1084-1092.

Durrant JD, Sabo DL, Hyre RJ (1990) Gender, head size, and ABRs examined in large clinical sample. Ear Hear 11:210-214.

Engineer ND, Riley JR, Seale JD, Vrana WA, Shetake JA, Sudanagunta SP, Borland MS, Kilgard MP (2011) Reversing pathological neural activity using targeted plasticity. Nature 470:101-104.

Finlayson PG, Kaltenbach JA (2009) Alterations in the spontaneous discharge patterns of single units in the dorsal cochlear nucleus following intense sound exposure. Hear Res 256:104-117.

Henry JA, Dennis KC, Schechter MA (2005) General review of tinnitus: prevalence, mechanisms, effects, and management. J Speech Lang Hear Res 48:1204-1235.

Hoffman HJ, Reed GW (2004) Epidemiology of tinnitus. In: Tinnitus: Theory and management (Snow JB, ed), pp 16-41. Hamilton, Ontario: Decker.

Houweling AR, Bazhenov M, Timofeev I, Steriade M, Sejnowski TJ (2005) Homeostatic synaptic plasticity can explain post-traumatic epileptogenesis in chronically isolated neocortex. Cereb Cortex 15:834-845.

Jacobson M, Kim S, Romney J, Zhu X, Frisina RD (2003) Contralateral suppression of distortion-product otoacoustic emissions declines with age: a comparison of findings in CBA mice with human listeners. Laryngoscope 113:1707-1713.

Kaltenbach JA (2011) Tinnitus: Models and mechanisms. Hear Res 276:52-60.

Kilman V, van Rossum MC, Turrigiano GG (2002) Activity deprivation reduces miniature IPSC amplitude by decreasing the number of postsynaptic $\mathrm{GABA}_{\mathrm{A}}$ receptors clustered at neocortical synapses. J Neurosci 22:1328-1337.

Kim S, Frisina DR, Frisina RD (2002) Effects of age on contralateral suppression of distortion product otoacoustic emissions in human listeners with normal hearing. Audiology and neuro-otology 7:348-357.

König O, Schaette R, Kempter R, Gross M (2006) Course of hearing loss and occurrence of tinnitus. Hear Res 221:59-64.

Kotak VC, Fujisawa S, Lee FA, Karthikeyan O, Aoki C, Sanes DH (2005) Hearing loss raises excitability in the auditory cortex. J Neurosci 25:3908-3918.

Kujawa SG, Liberman MC (2009) Adding insult to injury: cochlear nerve degeneration after "temporary" noise-induced hearing loss. J Neurosci 29:14077-14085.

Meddis R (2006) Auditory-nerve first-spike latency and auditory absolute threshold: a computer model. J Acoust Soc Am 119:406-417.

Moller AR (2007) Neural generators for auditory brainstem evoked potentials. In: Auditory evoked potentials (Burkard RF, Don M, Eggermont JJ, eds), pp 336-354. Baltimore: Lippincott Williams and Wilkins.

Morgenstern L (2005) The bells are ringing: tinnitus in their own words. Perspect Biol Med 48:396-407.

Mulders WH, Robertson D (2009) Hyperactivity in the auditory midbrain after acoustic trauma: dependence on cochlear activity. Neuroscience 164:733-746.

Norena A, Micheyl C, Chéry-Croze S, Collet L (2002) Psychoacoustic characterization of the tinnitus spectrum: implications for the underlying mechanisms of tinnitus. Audiol Neurootol 7:358-369.

Parra LC, Pearlmutter BA (2007) Illusory percepts from auditory adaptation. J Acoust Soc Am 121:1632-1641.

Rauschecker JP, Leaver AM, Mühlau M (2010) Tuning out the noise: limbic-auditory interactions in tinnitus. Neuron 66:819-826.

Roberts LE, Eggermont JJ, Caspary DM, Shore SE, Melcher JR, Kaltenbach JA (2010) Ringing ears: the neuroscience of tinnitus. J Neurosci 30:14972-14979.

Sanchez TG, Medeiros IR, Levy CP, Ramalho Jda R, Bento RF (2005) Tinnitus in normally hearing patients: clinical aspects and repercussions. Braz J Otorhinolaryngol 71:427-431. 
Schaette R, Kempter R (2006) Development of tinnitus-related neuronal hyperactivity through homeostatic plasticity after hearing loss: a computational model. Eur J Neurosci 23:3124-3138.

Schaette R, Kempter R (2008) Development of hyperactivity after hearing loss in a computational model of the dorsal cochlear nucleus depends on neuron response type. Hear Res 240:57-72.

Schaette R, Kempter R (2009) Predicting tinnitus pitch from patients' audiograms with a computational model for the development of neuronal hyperactivity. J Neurophysiol 101:3042-3052.

Stephens SD (1984) The treatment of tinnitus-a historical perspective. J Laryngol Otol 98:963-972.

Turrigiano GG (1999) Homeostatic plasticity in neuronal networks: the more things change, the more they stay the same. Trends Neurosci 22:221-227.

Turrigiano GG, Leslie KR, Desai NS, Rutherford LC, Nelson SB (1998) Activity-dependent scaling of quantal amplitude in neocortical neurons. Nature 391:892-896.
Vale C, Sanes DH (2002) The effect of bilateral deafness on excitatory and inhibitory synaptic strength in the inferior colliculus. Eur J Neurosci $16: 2394-2404$.

Weisz N, Hartmann T, Dohrmann K, Schlee W, Norena A (2006) Highfrequency tinnitus without hearing loss does not mean absence of deafferentation. Hear Res 222:108-114.

Whiting B, Moiseff A, Rubio ME (2009) Cochlear nucleus neurons redistribute synaptic AMPA and glycine receptors in response to monaural conductive hearing loss. Neuroscience 163:1264-1276.

Zettel ML, Zhu X, O’Neill WE, Frisina RD (2007) Age-related decline in Kv3.1b expression in the mouse auditory brainstem correlates with functional deficits in the medial olivocochlear efferent system. J Assoc Res Otolaryngol 8:280-293.

Zhu X, Vasilyeva ON, Kim S, Jacobson M, Romney J, Waterman MS, Tuttle D, Frisina RD (2007) Auditory efferent feedback system deficits precede age-related hearing loss: contralateral suppression of otoacoustic emissions in mice. J Comp Neurol 503:593-604. 